\title{
AN ANALYSIS OF EFFECTIVE THERMAL CONDUCTIVITY OF HETEROGENEOUS MATERIALS
}

\author{
Guocheng Zhu' ${ }^{1 *}$, Dana Kremenakova', Yan Wang ${ }^{1}$, Jiri Militky¹, Funda Buyuk Mazari²
}

1Department of textile material engineering, Technical University of Liberec, Studentská 1402/2, Liberec, Czech Republic,

2Department of clothing technology, Technical University of Liberec, Studentská 1402/2, Liberec, Czech Republic Email: zgc100100@hotmail.com

\begin{abstract}
:
Effective thermal conductivity (ETC) is a very important index for evaluating the thermal property of heterogeneous materials, which include more than two different kinds of materials. Several analytical models were proposed for predicting the ETC of heterogeneous materials, but in some cases, these models cannot provide very accurate predictions. In this work, several analytical models and numerical simulations were studied in order to investigate the differences among them. In addition, some factors which would influence the ETC of heterogeneous materials were investigated by numerical simulation. The results demonstrated that the numerical simulation can provide very accurate prediction, indicated that different analytical models should be selected to predict specific problems based on their assumptions, and suggested that more variables need to be considered in order to improve these analytical models, such as inclusion shape, inclusion size, distribution of inclusions and contact area. Besides, numerical method could be an effective and reliable way to obtain the ETC of heterogeneous materials with any kind of complicated structures.
\end{abstract}

\section{Keywords:}

Effective thermal conductivity, heterogeneous material, analytical model, numerical method

\section{Introduction}

Thermal conductivity (TC) of a material can be defined as the rate of heat transfer through a unit thickness of the material per unit area per unit temperature difference [1], which is one of the basic transport properties that significantly affects heat transfer and clothing comfort. Plenty of work has been done and is still going on to understand the phenomenon of steady-state heat transfer in general and through textiles and porous materials in particular. Ismail et al. [2] developed a mathematical model for woven fabrics with simple structure, which determines the effective thermal conductivity (ETC) of woven fabric by assuming that the gas phase is stagnant. Bhattacharjee and Kothari [3] proposed a model for prediction of thermal resistance of woven fabrics by using thermal electrical analogy technique. Yoshihiro et al. [4] developed structural models of yarns, plain weave fabrics and plain weave fabric/resin composites and theoretical formulas for the ETC which were based on the thermal resistance network analysis and volume fraction. Zhu and Li [5] developed a fractal ETC model for woven fabrics with multiple layers. Das et al. [6] developed a mathematical model for prediction of thermal resistance of multilayer clothing in non-convective media. Matusiak [7] developed a model of thermal resistance of woven fabrics and considered cross-section of yarn as square shape. But the prediction models for special-shaped fibres and yarns are missing. On the other hand, it is very difficult to measure the TC of a single fibre since it has a very small diameter and is very flexible. Though a few measurements which evaluated a composite specimen including a bundle of the fibres have been reported, these methods sometimes give TC values far more than the value for a single fibre [8-10].
Special-shaped fibres and textiles which contain fibre material and air can be taken as porous material or heterogeneous material. Therefore, the models of ETC of porous materials were used in the textiles. For heterogeneous materials with simple structure, the fundamental principle like thermal resistance networks can be used to obtain the ETC of heterogeneous materials. However, for heterogeneous materials with complicated physical structures, it is not possible to give the thermal resistance networks, and then some empirical analytical models were reported. Bogaty et al. [11] proposed one predicting model for textiles by combining series structure model and parallel structure model, and gave one coefficient for each component, but these coefficients would be changed when the arrangement of textiles is different; therefore, this model cannot be widely used. Faleh [12] stated one polynomial predicting model which needs to get the coefficient from every practical experiment for evaluating the TC of fibre in composites. Militky and Kremenakova [13] simplified the predicting model for ETC of textiles by getting the average value of the series and parallel models. The Maxwell-Eucken model $[14,15]$ was used for materials with continuous and dispersed phases. It assumes a dispersion of small spheres within a continuous matrix of a different component, with the spheres being far enough apart so that the local distortions to the temperature distributions around each of the spheres do not interfere with their neighbours' temperature distributions. Wang et al [16] deduced a mathematical expression for co-continuous structural materials based on Maxwell-Eucken model, but his model also can be expressed by the series and parallel models. Levy [17] gave a model based on the Maxwell-Eucken model, but Levy's model was derived solely by algebraic manipulation, with no stated physical basis. 
On the other hand, the numerical method has been widely used in every area due to its reliable accuracy, flexibility for both realistic conditions and ideal conditions, and more detailed information. Carson et al. [18] and Dasgupta et al. [19] stated that the numerical simulation for heat transfer had good agreement with the experimental results. The aim of this work is to compare the results from these analytical models and numerical simulation with the results based on Fourier's law, and to investigate some factors which have an effect on ETC of heterogeneous materials.

\section{Method}

In order to compare the accuracy and difference of analytical models and numerical simulation, heterogeneous materials with different inclusion shapes, volume fractions, inclusion size, distribution, cross-section length of inclusion and contact area were adopted. From the analytical model, only one group data would be provided even if so many different factors were adopted in the experiments since these analytical models only take the volume fraction into consideration. But numerical simulation can provide more specific results according to different parameters. Heterogeneous materials include two kinds of materials with TC of $k 1\left(=0.4 \mathrm{~W} \cdot \mathrm{m}^{-1} \cdot \mathrm{K}^{-1}\right)$ and $k 2(=1$ $\left.\mathrm{W} \cdot \mathrm{m}^{-1} \cdot \mathrm{K}^{-1}\right)$, which can be any kind of materials for the aim is to compare the accuracy and difference of analytical models and numerical simulation.

\section{Fourier's law of heat conduction}

Fourier's law is based on some assumptions, like perfect boundaries among materials, perfect geometrical shapes, and stagnant conditions of the gas/liquid phases, no humidity and no moisture absorption effects.

\section{Thermal resistance networks}

Thermal resistance network is one effective method to analyse TC of heterogeneous materials with simple structures which can be easily divided into several zones. The concept of thermal resistance network is analogous to the electrical resistance concept. Therefore, the ETC of heterogeneous materials can be obtained by the combinations of TC of each zone. One simple example is given in Figure 1.
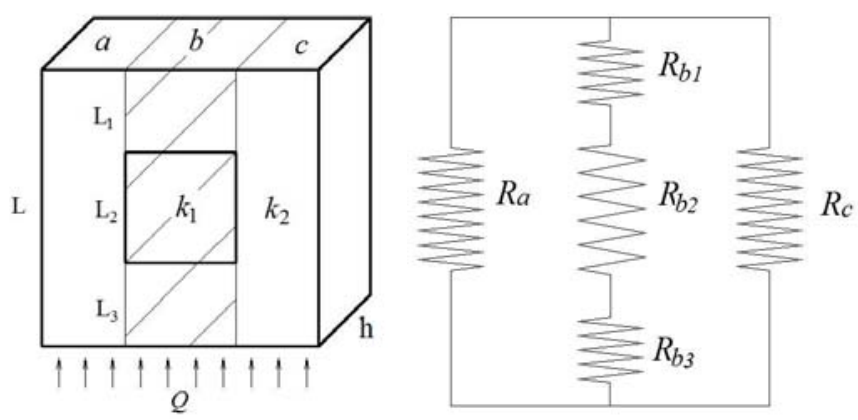

Figure 1. Left: structure of one heterogeneous material; right: the corresponding thermal resistance networks
The following equations are used for calculating the ETC of heterogeneous material with square and rectangular inclusions.

$$
\begin{aligned}
& R_{a}=\frac{L}{a \cdot h \cdot k_{2}} \\
& R_{b}=R_{b 1}+R_{b 2}+R_{b 3} ; \quad R_{b 1}=\frac{L_{1}}{b \cdot h \cdot k_{2}}, \\
& R_{b 2}=\frac{L_{2}}{b \cdot h \cdot k_{1}}, \quad R_{b 3}=\frac{L_{3}}{b \cdot h \cdot k_{2}} \\
& R_{c}=\frac{L}{c \cdot h \cdot k_{2}} \\
& R_{t o t}=\frac{R_{a} R_{b} R_{c}}{R_{a} R_{b}+R_{a} R_{c}+R_{b} R_{c}} \\
& k_{e}=\frac{L}{A \cdot R_{t o t}}
\end{aligned}
$$

where $k 1$ and $k 2$ are TC of material 1 and material 2 ; ke represents ETC of heterogeneous material $\left(\mathrm{W} \cdot \mathrm{m}^{-1} \cdot \mathrm{K}^{-1}\right)$; $R a$, $R b, R c$ and $R$ tot represent thermal resistance of zones $a, b, c$ and total thermal resistance (K/W); $A$ is area where heat flow goes through $\left(\mathrm{m}^{2}\right) ; L$ is height of material $(\mathrm{m}) ; a, b$ and $c$ are the length of material in zones $a, b$ and $c$, respectively $(\mathrm{m})$; and $h$ is the width of material $(\mathrm{m})$.

\section{Heat flow by infinitesimal method}

Unfortunately, it is not easy to distinguish the sub-regions of a heterogeneous material such as circular, elliptic or other irregular inclusions; therefore, the differential concept was adopted. Generally speaking, this method is the extension of the thermal resistance networks, but the motivation of this method is to consider the heat flow through the objective first. The geometrical model is divided into infinitesimal area (Figure 2), the heat flow from each infinitesimal area is accumulated and then the ETC is calculated by Fourier's equation. In this paper,

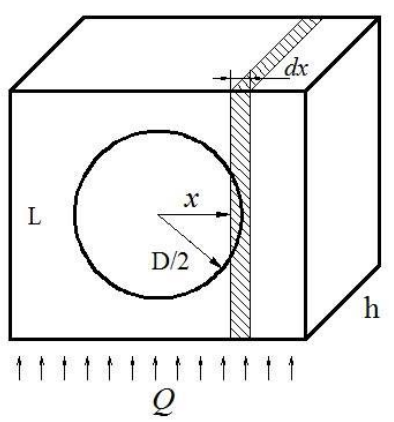

Figure 2. The geometrical model of heterogeneous material including circular inclusion 
only the circular and elliptic inclusions were considered to get rid of the difficulties from very irregular inclusions. Owing to the symmetry of the geometrical model, only half-a-part was taken into consideration. The heat flow and ETC can be obtained from following equations.

The heat flow that goes through a material can be divided into two parts: Q1, heat flow goes through both inclusion and covering material $(\mathrm{W} \cdot \mathrm{s})$; $\mathrm{Q} 2$, heat flow only goes through covering material $(\mathrm{W} \cdot \mathrm{s})$.

$$
\begin{gathered}
\frac{Q_{1}}{2}=\int_{0}^{\frac{D}{2}} \Delta T \cdot h \cdot d x /\left[\left(L-2 \sqrt{(D / 2)^{2}-x^{2}} / k_{2}\right)+\left(2 \sqrt{(D / 2)^{2}-x^{2}} / k_{1}\right)\right] \\
\frac{Q_{2}}{2}=\int_{D / 2}^{L / 2} \frac{\Delta T \cdot h \cdot d x}{L / k_{2}} \\
k_{e}=\frac{Q \cdot L}{A \cdot \Delta T},\left(Q=Q_{1}+Q_{2}\right)
\end{gathered}
$$

where $L$ and $A$ are distance $(\mathrm{m})$ and area $\left(\mathrm{m}^{2}\right)$ of heat flow that goes through, $D$ is diameter of circular inclusion $(\mathrm{m}), h$ is thickness of heterogeneous material $(\mathrm{m}), \Delta \mathrm{T}$ is temperature difference $(\mathrm{K})$, and $k 1$ and $k 2$ are the TC of inclusion material and the covering material $\left(\mathrm{W} \cdot \mathrm{m}^{-1} \cdot \mathrm{K}^{-1}\right)$. In equation (6), the first term in the square bracket on the right side represents the value of covering material fraction divided by the TC of covering material, which means the thermal resistance of covering material. Similarly, the second term in the square bracket on the right side represents the thermal resistance of inclusion material. Therefore, the sum of these two terms is the total thermal resistance of the heterogeneous material in each infinitesimal area. The integrand is another form of Fourier's equation.

Similarly, the expressions for ETC of heterogeneous material including elliptic inclusion are:

$$
\frac{Q_{1}}{2}=\int_{0}^{a} \Delta T \cdot h \cdot d x /\left[\left(L-2 \sqrt{b^{2}-b^{2} x^{2} / a^{2}} / k_{2}\right)+\left(2 \sqrt{b^{2}-b^{2} x^{2} / a^{2}} / k_{1}\right)\right]
$$

$$
\begin{gathered}
\frac{Q_{2}}{2}=\int_{a}^{L / 2} \frac{\Delta T \cdot h \cdot d x}{L / k_{2}} \\
k_{e}=\frac{Q \cdot L}{A \cdot \Delta T}
\end{gathered}
$$

where $a$ is the length of major semi-axis in $x$ direction $(\mathrm{m})$ and $b$ is the length of minor semi-axis in $y$ direction $(\mathrm{m})$.

\section{Some analytical models}

Some analytical models for predicting ETC of heterogeneous materials are shown in Table 1. The first two models are widely used for heterogeneous materials containing continuous phases such as textile materials. The last two models are usually used for heterogeneous materials containing particles.

Table 1. Some ETC models of heterogeneous materials

\begin{tabular}{|c|c|c|}
\hline Models & Effective thermal conductivity equation & References \\
\hline Series-parallel 1 & $k_{e}=\left(k_{P}+k_{S}\right) / 2$ & \\
& $k_{P}=P k_{1}+(1-P) k_{2}$ & [13] \\
\hline Series-parallel 2 & $k_{s}=\frac{k_{1} k_{2}}{P k_{2}+(1-P) k_{1}}$ & \\
\hline Maxwell-Eucken & $k_{e}=k_{s} / 2\left(\sqrt{1+8 k_{p} / k_{s}}-1\right)$ & [16] \\
\hline & $k_{p}=\sum_{i=1}^{N} k_{i} v_{i}, k_{s}=1 / \sum_{i=1}^{N} \frac{v_{i}}{k_{i}}$ & [14, 15] \\
\hline Levy's & $k_{e}=k_{1} \frac{2 k_{1}+k_{2}-2\left(k_{1}-k_{2}\right) v_{2}}{2 k_{1}+k_{2}+\left(k_{1}-k_{2}\right) v_{2}}$ & \\
& $k_{e}=k_{1} \frac{2 k_{1}+k_{2}-2\left(k_{1}-k_{2}\right) F}{2 k_{1}+k_{2}+\left(k_{1}-k_{2}\right) F}$ & [17] \\
\hline
\end{tabular}


The textile material is described as a fibre-air mixture or heterogeneous material. In these models, $P$ is volume fraction of material $1, k e$ is ETC of heterogeneous material, $k p$ and $k s$ are TC of materials with parallel arrangement and series arrangement, $k i$ and $v i$ are the TC and volume fraction of ith material, $v 1$ and $v 2$ are the volume fraction of materials 1 and 2 , and $k 1$ and $k 2$ are the TC of materials 1 and 2 .

\section{Numerical simulation}

Numerical methods include finite difference methods, boundary element methods and finite element methods which have the advantage that the discretization process is not constrained by regular node positioning, and they are therefore more suitable for irregular geometries and for spatially variable properties and conditions.

Therefore, the finite element models were set up to simulate a three-dimensional steady-state TC measurement apparatus. One geometrical model and grid topologies of simulations are given in Figure 3 . The boundary conditions imposed on the model faces were: one side was kept at constant temperature while its opposite side was subjected to heat flow; the other two sides were kept thermally insulated. Hence, after getting temperature distribution of heterogeneous material, the ETC can be calculated from equation (8).
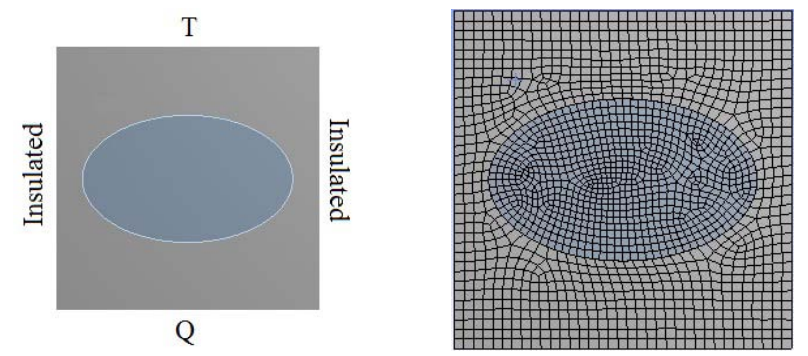

Figure 3. Geometrical model and grid topology

And the governing equation used in numerical simulation is:

$$
[C(T)]\{\dot{T}\}+[K(T)]\{T\}=[Q(T)]
$$

where $\{T\}$ represents temperature matrix, $[C]$ represents specific heat matrix, $[K]$ represents thermal conduction matrix, $[Q]$ represents heat flux vector matrix and $\{\dot{T}\}$ is the derivative of the temperature with respect to time.

Therefore, the equation for steady-state thermal analysis is given by:

$$
[K]\{T\}=\{Q\}
$$

where $\{Q\}$ is the heat flux vector at nodes.

Simulation 1. Four types of inclusion shapes - square (SI), rectangle $(\mathrm{RI})$, circle $(\mathrm{Cl})$ and ellipse $(\mathrm{EI})$ - were investigated and the volume fractions of inclusions were from $10 \%$ to $70 \%$.

Simulation 2. The inclusion sizes were from $0.12 \%$ to $30 \%$ of total size of heterogeneous material, and the inclusion volume fraction was kept constant.

Simulation 3. Six distributions of inclusions were investigated, and the inclusion volume fraction was kept constant. The circles represent fibres, and the rest represents another material.

Simulation 4. The major semi-axes of elliptic inclusions were from $41.52 \%$ to $95.49 \%$ of the total cross-section length of heterogeneous materials, and the inclusion volume fraction was kept constant.

Simulation 5. The contact length/area between inclusions was from $0 \%$ to $63 \%$ of inclusion length, and the inclusion volume fraction was kept constant.
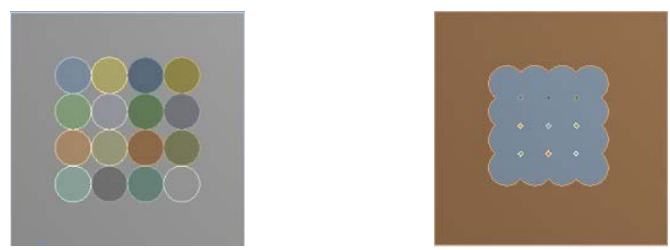

Figure 6. Different contact length/area between inclusions
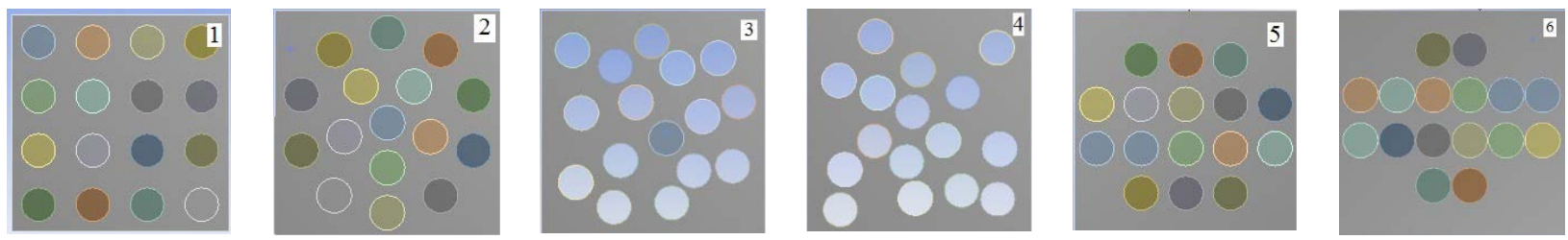

Figure 4. Different distributions of inclusions


Figure 5. Different major semi-axes of elliptic inclusions 


\section{Results and discussion}

\section{Comparison of results from some models and results based on Fourier's law}

Comparing with the results based on Fourier's law, analytical models would be less accurate since these models were either based on some assumptions or on experimental results. During the experimental process, there are serious difficulties of measurement in many situations, and the measuring instruments are not free from errors. Therefore, the results based on Fourier's law were taken as reference values and the accuracy of analytical models was checked by comparing with the reference values. In order to evaluate the accuracy of analytical models easier, deviation was adopted (Table 2). The deviation, $\mathrm{De}$, can be obtained from the following equation:

$$
D_{e}=\left|\frac{k^{\prime}-k_{F}}{k_{F}}\right|
$$

where $k$ ' represents the TC from models or numerical simulation and $k F$ represents the TC based on Fourier's law.

The ETC from analytical models and based on Fourier's law had a slight difference at each volume fraction, and the ETC decreased as volume fraction of inclusions increased owing to the lower TC of inclusion (Figure 7). The deviations of ETC from analytical models are shown in Table 2. Every analytical model has its advantages and limitations due to assumptions. Based on this work, PSM1 has better predicting accuracy than the other models. The results from PSM1 provided the smallest deviation when the inclusion shapes were square, circle and ellipse compared with the results from other models, especially when the inclusion shape was square. The reason could be that the geometrical model is regular and easy to be divided into bulk sub-regions, which is more close to the thermal resistance networks analysis method. ME model is usually derived from a specific assumed physical structure as mentioned above, but it can also provide results which have good agreement with the results based on Fourier's law. PSM2 extended the application of ME model to co-continuous structure heterogeneous materials, but it did not improve the accuracy of ME model since all assumptions were based on ME model. Levy's model added one coefficient to ME model, which in some conditions can improve the accuracy of predicting results. Moreover, the accuracy of ETC from analytical models was influenced by inclusion shapes. For PSM1 and ME, they have better predicting results for square and circular inclusions than rectangular and elliptic inclusions; for PSM2 and LM, the better predicting results were for square and rectangular inclusions. Some researchers stated that the prediction of the $\mathrm{TC}$ of heterogeneous materials has proven to be a difficult task. Part of the difficulty may be due to an over-simplification of the structure [20].

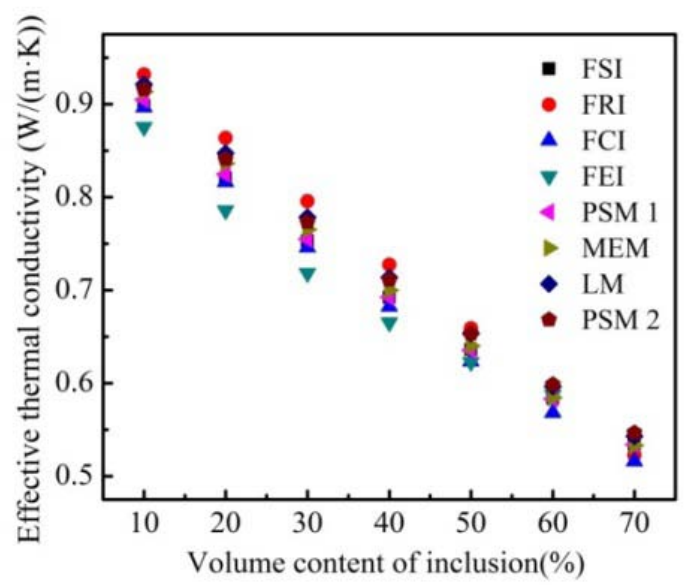

Figure 7. Comparison of results from some models and results based on Fourier's law

FSI: results of square inclusions based on Fourier's law; FRI: results of rectangular inclusions based on Fourier's law; FCl: results of circular inclusions based on Fourier's law; FEI: results of elliptic inclusions based on Fourier's law; PSM1: parallel-series model 1; MEM: Maxwell-Eucken model; LM: levy's model; PSM2: parallelseries model 2

\section{Comparison of results from numerical simulation and results based on Fourier's law}

The ETC of heterogeneous materials from numerical simulation and results based on Fourier's law are given in Figure 8. As mentioned above, the results based on Fourier's law were taken as reference values. And then the accuracy of numerical simulation was checked by comparing with the reference values. The maximum deviation and mean deviation of ETC from numerical simulation are given in Table 3. Heterogeneous material including rectangular inclusions gave the biggest deviation, and the circular inclusions provided the smallest deviation. Generally, the deviations from numerical simulations were less than $3 \%$, which provided enough accurate results. On the other hand, comparing with the analytical models, the numerical simulation is suitable for any type of inclusion shapes.

Table 2. Deviation of ETC from analytical models

\begin{tabular}{|c|c|c|c|c|c|c|c|c|}
\hline \multirow[b]{2}{*}{$\begin{array}{l}\text { Inclusion } \\
\text { shape }\end{array}$} & \multicolumn{2}{|c|}{ Parallel-series model 1} & \multicolumn{2}{|c|}{ Parallel-series model 2} & \multicolumn{2}{|c|}{ ME model } & \multicolumn{2}{|c|}{ Levy's model } \\
\hline & $\begin{array}{c}\text { Maximum } \\
\text { deviation } \\
(\%)\end{array}$ & $\begin{array}{c}\text { Mean } \\
\text { deviation } \\
(\%)\end{array}$ & $\begin{array}{c}\text { Maximum } \\
\text { deviation } \\
(\%)\end{array}$ & $\begin{array}{c}\text { Mean } \\
\text { deviation } \\
(\%) \\
\end{array}$ & $\begin{array}{c}\text { Maximum } \\
\text { deviation } \\
(\%)\end{array}$ & $\begin{array}{c}\text { Mean } \\
\text { deviation } \\
(\%) \\
\end{array}$ & $\begin{array}{c}\text { Maximum } \\
\text { deviation } \\
(\%)\end{array}$ & $\begin{array}{c}\text { Mean } \\
\text { deviation } \\
(\%)\end{array}$ \\
\hline Square & 0.9 & 0.24 & 2.69 & 2.48 & 1.94 & 1.05 & 3.37 & 2.68 \\
\hline Rectangle & 4.24 & 3.46 & 4.63 & 2.34 & 3.81 & 2.65 & 3.86 & 1.83 \\
\hline Circle & 3.47 & 1.83 & 5.99 & 4.19 & 3.35 & 2.67 & 5.21 & 4.38 \\
\hline Ellipse & 3.08 & 3.39 & 7.64 & 5.49 & 6.54 & 4.32 & 8.38 & 5.86 \\
\hline
\end{tabular}


Table 3. Deviation of ETC from numerical simulation

\begin{tabular}{|c|c|c|c|c|}
\hline Inclusion shape & Square & Rectangle & Circle & Ellipse \\
\hline $\begin{array}{c}\text { Maximum } \\
\text { deviation (\%) }\end{array}$ & 2.13 & 4.24 & 1.78 & 3.08 \\
\hline $\begin{array}{c}\text { Mean deviation } \\
(\%)\end{array}$ & 1.22 & 2.95 & 1.09 & 1.5 \\
\hline
\end{tabular}

\section{Effect of inclusion shape on ETC}

In this article, we have been discussing that the inclusion shape has the potential to influence the ETC of heterogeneous materials [20,21]. In this work, four types of inclusion shapes - square, rectangle, circle and ellipse - at different volume fraction were investigated based on Fourier's law of heat conduction. For rectangular and elliptic inclusions, the dimensions are also related to the inclusion shapes even though the volume fractions could be the same. Therefore, one side length of rectangular inclusion and the major semi-axis of elliptic inclusion were kept as a constant which was $80 \%$ of heterogeneous material's side length. The heterogeneous material including elliptic inclusions gave the smallest ETC. Square and circular inclusions in heterogeneous materials showed very similar results, which are the same as with the work of Carson et al. [18]. Rectangular inclusions showed the biggest ETC, which does not have an agreement with the result of Carson et al. [18], because in their work, the dimension of rectangular inclusions were set as $2: 1$, and these inclusions were distributed randomly. These different results indicated that the dimensions of inclusion also could be one factor which would influence ETC of heterogeneous materials. Comparing with the ETC of heterogeneous materials including different inclusion shapes with the same volume fraction, the biggest difference can reach $10.67 \%$ (Figure 9), which reveals that the inclusion shape is a significant factor.

\section{Effect of inclusions size on ETC}

Some researchers $[18,20]$ stated that the effect of porosity on TC cannot be described solely by volume fraction. Carson et al. [18] reported that the ETC of heterogeneous materials with different inclusion sizes were similar over the range of volume fractions. In this work, the ETC decreased obviously as the increase of inclusion size (Figure 10). The possible reason is that the small inclusions increased the interface area, which is helpful for dissipating heat when they contacted with better conductor.

\section{Effect of distribution of inclusions on ETC}

Generally, the distribution of inclusions has the same effect with the inclusion shape since different combination of small inclusions can form different structures of a larger inclusion. The difference between the largest and smallest ETC was $6.3 \%$. Comparing with these results corresponding to the distribution, the smallest ETC happened when more inclusions are at cross-section (distribution 6), and the opposite case is distribution 1. In order to confirm the conclusion that the inclusion length ratio in cross-section would influence ETC,

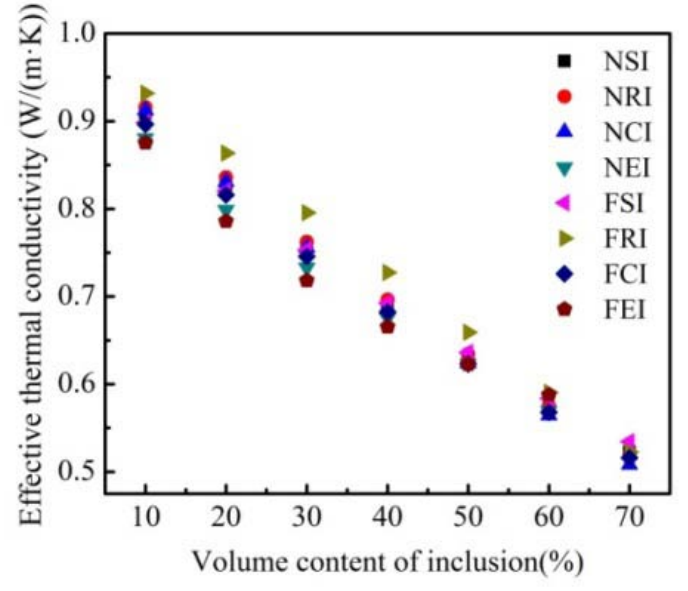

Figure 8. Comparison of ETC from numerical simulation and results based on Fourier's law

NSI: results of square inclusions from numerical simulation; NRI: results of rectangular inclusion from numerical simulation; $\mathrm{NCl}$ : results of circular inclusions from numerical simulation; NEl: results of elliptic inclusions from numerical simulation

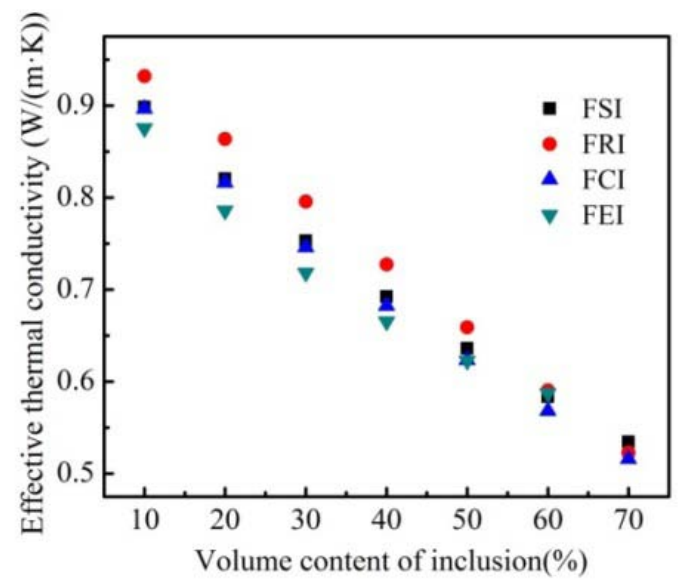

Figure 9. ETC of heterogeneous material including different inclusion shapes

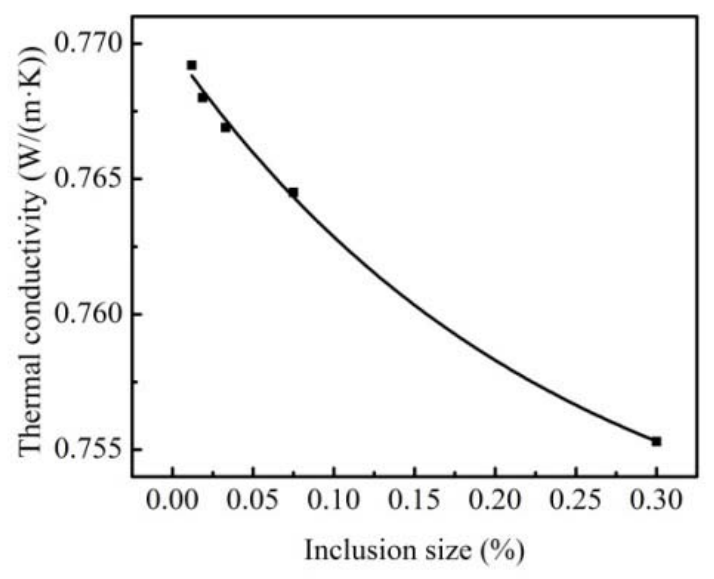

Figure 10. Effect of inclusion size on ETC of heterogeneous material 
one more simulation focusing on the cross-section length of inclusion was carried out subsequently.

\section{Effect of cross-section length of inclusion on ETC}

Based on the conception of parallel and series structures, the cross-section length of inclusions should have an effect on ETC of heterogeneous material. Therefore, elliptic inclusions with different dimensions at the same volume content were investigated. The results showed that ETC was inversely proportional to the cross-section length of inclusions, and the biggest difference reached $8.45 \%$.

\section{Effect of contact length/area among inclusions on ETC}

Heat conduction happens only when materials contact with each other, and the larger the contact area, the faster the heat conduction will be. The results suggested that the contact had a significant effect on ETC, and the biggest deviation was $5.41 \%$ which was not a very large difference due to the small difference of TC of these two materials. This simulation also can be used to analyse the ETC of yarns with different packing density.

\section{Conclusions}

Effective TC of some heterogeneous materials with simple structure was investigated by analytical models and numerical simulation in this work. Significant conclusions drawn from the results are: (1) analytical models presented in this paper can provide accurate prediction and have a very small difference among them; (2) numerical simulation can provide more accurate results than analytical models and is more flexible for any structure; besides, numerical simulation can be a good way to evaluate ETC of textiles with complicated structures and experimental conditions due to the difficulties in experiment; and (3) more important parameters need to be considered for improving analytical model, such as inclusion of shapes, the length ratio of inclusions in cross-section and contact area.

\section{Acknowledgement}

This work is supported under Student Grant Scheme (SGS 48013/115) by Technical University of Liberec, Czech Republic.

\section{References}

[1] Cengel, Y. A., (2003). Heat transfer: A practical approach. (2nd ed). New York, McGraw-Hill.

[2] Ismail, M. I.; Ammar, A. S. A.; Elokeily, M. (1988). Heat Transfer through Textile Fabrics: Mathematical Model. Appl Math Model, 12 (4), 434-440.

[3] Bhattacharjee, D.; Kothari, V. K. (2009). Heat transfer through woven textiles. Int $J$ Heat Mass Tran,52 (7-8), 2155-2160.

[4] Ymashita Yoshihiro; Yamda Hiroakia; Hajimeb, M. (2008). Effective Thermal Conductivity of Plain Weave Fabric and its Composite Material Made from High Strength Fibers Journal of Textile Engineering, 54 (4), 111-119.

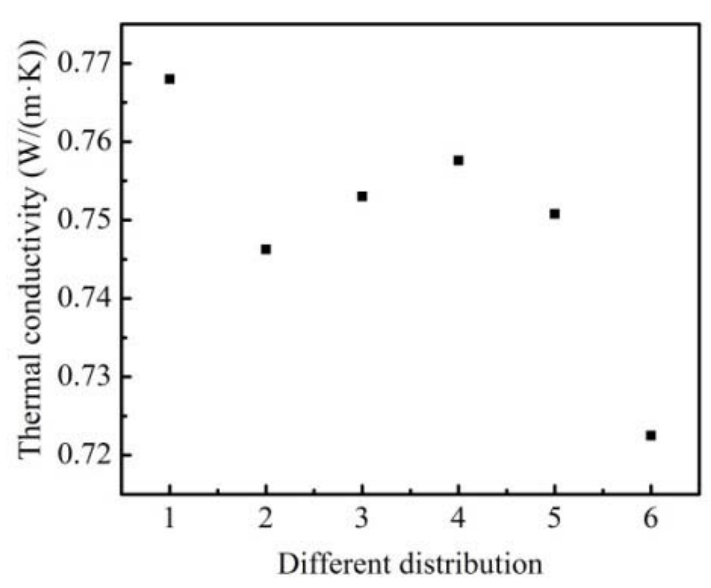

Figure 11. Effect of distribution on ETC of heterogeneous material

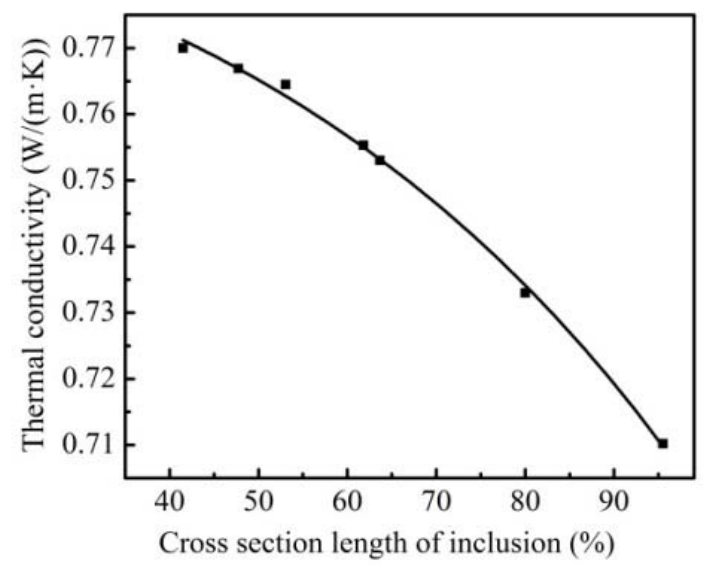

Figure 12. Effect of cross-section length of inclusion on ETC of heterogeneous material

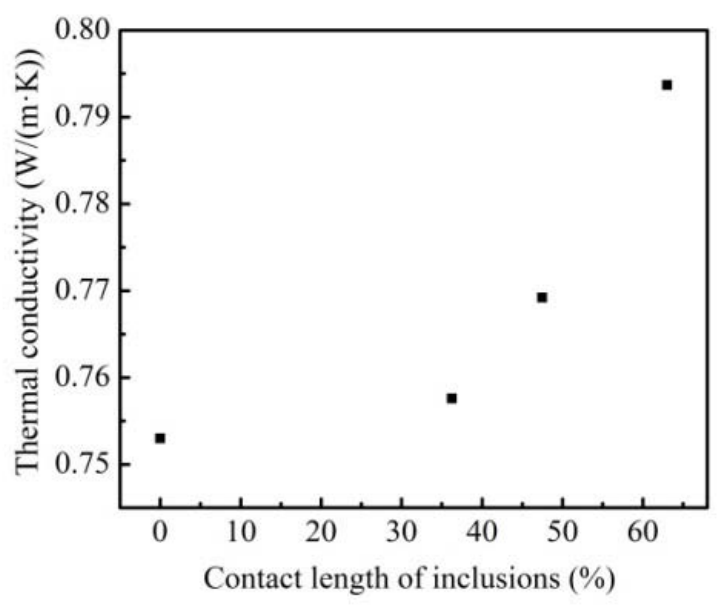

Figure 13. Effect of contact length among inclusions on ETC of heterogeneous material

[5] Zhu, F.; Li, K. (2010). Determining Effective Thermal Conductivity of Fabrics by Using Fractal Method. Int J Thermophys, 31 (3), 612-619.

[6] Das, A.; Alagirusamy, R.; Kumar, P. (2011). Study of heat transfer through multilayer clothing assemblies: $A$ theoretical prediction. Autex Res J,11 (2), 54-60. 
[7] Matusiak, M. (2012). Modelling the thermal resistance of woven fabrics. J Text I,104 (4), 426-437.

[8] Taylor, R. E.; Jortner, J.; Groot, H. (1985). ThermalDiffusivity of Fiber-Reinforced Composites Using the Laser Flash Technique. Carbon,23 (2), 215-222.

[9] Johnson, L. F.; Hasselman, D. P. H.; Chyung, K. (1987). Effect of Silicon-Carbide Fiber or Whisker Reinforcement on the Thermal-Diffusivity Conductivity of an Osumilite Glass-Ceramic. J Am Ceram Soc,70 (6), C135-C138.

[10] Zhang, X.; Fujiwara, S.; Fujii, M. (2000). Measurements of thermal conductivity and electrical conductivity of a single carbon fiber. Int J Thermophys, 21 (4), 965-980.

[11] Bogaty, H.; Hollies, N. R. S.; Harris, M. (1957). Some Thermal Properties of Fabrics: Part I: The Effect of Fiber Arrangement. Text Res J,27 (6), 445-449.

[12] Al-Sulaiman, F. A.; Al-Nassar, Y. N.; Mokheimer, E. M. A. (2006). Numerical prediction of the thermal conductivity of fibers. Heat Mass Transfer, 42 (5), 449-461.

[13] Jiri Militky; Kremenakova, D. In Prediction of fabrics thermal conductivity, 5th International textile, clothing \& design conference - Magic World of Textiles, Dubrovnik, Croatia, Dubrovnik, Croatia, 2010; pp 1-6.

[14] Eucken, A. (1940). Allgemeine Gesetzmabigkeiten fur das Warmeleitvermogen verschiedener Stoffarten und Aggregatzustande. Forschung Gabiete Ingenieur, 11 (1), 6-20.
[15] Maxwell, J. C., (1954). A Treatise on Electricity and Magnetism. (3rd ed). New York, Dover Publications Inc.

[16] Wang, J.; Carson, J. K.; North, M. F., et al. (2008). A new structural model of effective thermal conductivity for heterogeneous materials with co-continuous phases. Int $J$ Heat Mass Tran, 51 (9-10), 2389-2397.

[17] Levy, F. L. (1981). A modified Maxwell-Eucken equation for calculating the thermal conductivity of two-component solutions or mixtures. Int. J. Refrigeration, 4 (4), 223-225.

[18] Carson, J. K.; Lovatt, S. J.; Tanner, D. J., et al. (2003). An analysis of the influence of material structure on the effective thermal conductivity of theoretical porous materials using finite element simulations. Int J Refrig, 26 (8), 873-880.

[19] Dasgupta, A.; Agarwal, R. K.; Bhandarkar, S. M. (1996). Three-dimensional modeling of woven-fabric composites for effective thermo-mechanical and thermal properties. Compos Sci Technol,56 (3), 209-223.

[20] Krach, A.; Advani, S. G. (1996). Influence of void shape, void volume and matrix anisotropy on effective thermal conductivity of a three-phase composite. J Compos Mater, 30 (8), 933-946.

[21] Francl, J.; Kingery, W. D. (1954). Thermal Conductivity:IX, Experimental Investigation of Effect of Porosity on Thermal Conductivity. J Am Ceram Soc,37 (2), 99-107. 